\title{
Self-dual solitons in a generalized Chern-Simons baby Skyrme model
}

\author{
Rodolfo Casana, ${ }^{*}$ André C. Santos, ${ }^{\dagger}$ Claudio F. Farias, ${ }^{\ddagger}$ and Alexsandro L. Mota ${ }^{\S}$ \\ Departamento de Física, Universidade Federal do Maranhão, 65080-805 São Luís, Maranhão, Brazil
}

(Received 21 February 2019; published 26 August 2019)

\begin{abstract}
We have shown the existence of self-dual solitons in a type of generalized Chern-Simons baby Skyrme model in which the generalized function (depending only in the Skyrme field) is coupled to the sigmamodel term. The consistent implementation of the Bogomol'nyi-Prasad-Sommerfield formalism requires the generalizing function becomes the superpotential defining the self-dual potential properly. Thus, we have obtained a topological energy lower bound (Bogomol'nyi bound) and the self-dual equations satisfied by the fields saturating such a bound. The Bogomol'nyi bound being proportional to the topological charge of the Skyrme field is quantized, whereas the total magnetic flux is not. As expected in a Chern-Simons model, the total magnetic flux and the total electrical charge are proportional to each other. Thus, by considering the superpotential a well-behaved function in the whole target space, we have shown the existence of three types of self-dual solutions: compacton solitons, soliton solutions decaying by following an exponential-law $e^{-\alpha r^{2}}(\alpha>0)$, and solitons having a power-law decay $r^{-\beta}(\beta>0)$. The profiles of the two last solitons can exhibit compactonlike behavior. The self-dual equations have been solved numerically, and we have depicted the soliton profiles, commenting on the main characteristics exhibited by them.
\end{abstract}

DOI: 10.1103/PhysRevD.100.045022

\section{INTRODUCTION}

Effective field theories have an important role in physics, especially when they can provide answers or insights about certain physical properties that could be difficult or even impossible to extracted from the respective underlying higher-energy model. Among such effective models, the Skyrme model [1] was proposed with the aim to give some critical information about hadronic states, which result be a hard task when analyzed directly via Quantum Chromodynamics. The proposal of the Skyrme model is to substitute by means of a scalar triplet the Goldstone bosons produced by the chiral symmetry breaking [2]. Such an approach provides an efficient and very predictive framework for the study of baryon properties [3], as well as atomic nuclei [4], nuclear matter [5], and neutron stars [6]. The baryons emerge as collective excitations described by topological solitons called Skyrmions. Some modification into Skyrme's model can result in a better description of the baryon masses, such as that shown in Ref. [7].

\footnotetext{
*rodolfo.casana@gmail.com

†andre_cavs@hotmail.com

*cffarias@gmail.com

§lucenalexster@gmail.com
}

Published by the American Physical Society under the terms of the Creative Commons Attribution 4.0 International license. Further distribution of this work must maintain attribution to the author(s) and the published article's title, journal citation, and DOI. Funded by SCOAP .
The Skyrmions achieved a new status in physics when researchers found promising applications in condensed matter physics. Initially, they were studied in systems such as superfluid ${ }^{3} \mathrm{He}$ [8] and quantum Hall ferromagnets [9]. More recently, the discovery of Skyrmion structures in magnetic materials has been reported; for example, neutron scattering experiments have shown that a Skyrmion crystal configuration is related to phase transitions in a MnSi bulk [10], and Skyrmion behavior was found in Monte Carlo simulations running on a discretized model of the chiral magnet in two dimensions [11]. An important technological step was made when a Skyrmion phase was obtained on a thin film of the chiral magnet $\mathrm{Fe}_{1-x} \mathrm{Co}_{x} \mathrm{Si}$, which has energetic stability greater than in three-dimensional systems [12]. The research on magnetic Skyrmions is a promising area aiming for technological applications such as data storage and spintronics.

Recent developments were made on Bose-Einstein condensates [13] and chiral nematic liquid crystals [14]. There are also remarkable works on superconductivity. Skyrmions have been predicted for $\mathrm{K}_{2} \mathrm{Fe}_{4} \mathrm{Se}_{5}$ material in which superconductivity emerges at room temperature and stable Skyrmions become Cooper pairs through a quantum anomaly [15]. Other approaches involving analogies between vortex in superconductors systems and Skyrmions in magnetic materials has been investigated in Refs. [16-20]. A Skyrmion crystal with a triangular array in magnetic systems was shown to have strong similarities with the Abrikosov vortex lattice in type-II superconductors [21]. 
All these planar realizations and residual problems in the Skyrme approach on nuclear physics has inspired the development of a lower-dimensional version of the Skyrme model called the baby Skyrme model [22,23]. It can be seen as a toy model in $(2+1)$ dimensions that keeps some essential qualitative features of its higher-dimensional counterpart. The gauged versions of the baby Skyrme model have been built by introducing minimal covariant derivatives and the respective dynamical gauge term. Soliton solutions carrying only magnetic flux were obtained in a baby Skyrme model in which the gauge field dynamics is governed by Maxwell's term [24]. Until now, it has not been possible to implement the Bogomol'nyi-Prasad-Sommereld (BPS) formalism for the baby Skyrme model. However, the existence of BPS solitons in the gauged nonlinear sigma model [23,25] leads to an important conclusion: the lower bound of the full baby Skyrme model is not less than the sigma-model bound [25].

Nevertheless, the so-called restricted baby Skyrme model [26] possesses a BPS structure [27]. For the gauged version with the Maxwell term, the BPS solitons saturating the energy lower bound were finally found in Ref. [28]. In general, such models have shown themselves to be an interesting avenue of investigations in many issues such as duality between vortices and planar Skyrmions [29], topological phase transitions [30], the Bogomol'nyi equation from the strong necessary conditions [31], gauged BPS baby Skyrmions with quantized magnetic flux [32], supersymmetry [33-35], and gravitational theories [36].

In $(2+1)$ dimensions, besides the Maxwell term with its obvious relevance in gauged field theories, there is the topological Chern-Simons term, which has a central physical role in the emergence of configurations with non-null total electric charge. The Chern-Simons term plays an important role in field theory $[37,38]$ and in the description of some phenomena in bidimensional systems of condensed matter physics, such as fractional statistics [39] and the fractional quantum Hall effect [40]. In the context of topological defects involving the baby Skyrme model, the influence of the Chern-Simons term was studied in Ref. [41], which obtained soliton solutions with interesting new features such as electrical charge; Ref. [42] analyzed a Lifshitz version of a gauged baby Skyrme model providing BPS solitons; the multisoliton configurations and their changes under the influence of some potentials were studied in Ref. [43]. Recently, a supersymmetric extension was implemented in Ref. [44].

The goal of the manuscript is the successful implementation of the BPS formalism in a generalized version of a gauged baby Skyrme model in which the gauge field dynamics is governed solely by the Chern-Simons term. Such a model is able to engender BPS compacton and noncompacton solitons, and the latter ones can exhibit compactonlike behavior. The manuscript is structured as follows. In Sec. II, we present a Chern-Simons restricted baby Skyrme model in which the unsuccessful implementation of the BPS technique has allowed us to glimpse the guidelines for the construction of a model able to engender BPS configurations. In Sec. III, based on the previous section, we construct a true BPS Chern-Simons baby Skyrme model which allows us to obtain an energy lower bound and the respective self-dual or BPS equations. In Sec. IV, we analyze some properties of the rotationally symmetric solitons such as the behavior at boundaries, the magnetic flux, and electric charge. Section V is dedicated to the numerical solutions of the BPS equations. Finally, in Sec. VI, we present our conclusions and perspectives.

\section{NON-BPS CHERN-SIMONS RESTRICTED BABY SKYRME MODEL}

The baby Skyrme model $[22,45]$ is a $(2+1)$ dimensional nonlinear field theory supporting topological solitons described by the Lagrangian density

$$
\mathcal{L}=\frac{\lambda_{0}^{2}}{2} \partial_{\mu} \vec{\phi} \cdot \partial^{\mu} \vec{\phi}-\frac{\lambda^{2}}{4}\left(\partial_{\mu} \vec{\phi} \times \partial_{\nu} \vec{\phi}\right)^{2}-V .
$$

The first contribution represents the sigma-model term, the second one is the Skyrme term, and the third term is the self-interacting potential, the latter being (in principle) a function of the quantity $\hat{n} \cdot \vec{\phi}=\phi_{n}$, i.e., $V \equiv V\left(\phi_{n}\right)$. In the internal space, $\hat{n}$ is an unitary vector providing a preferred direction, and the Skyrme field $\vec{\phi}$ defines a triplet of real scalar fields $\vec{\phi}=\left(\phi_{1}, \phi_{2}, \phi_{3}\right)$ with fixed norm, $\vec{\phi} \cdot \vec{\phi}=1$, describing a spherical surface with unitary radius.

In the absence of the sigma-model term, the resulting one is the so-called restricted baby Skyrme model, which is given by

$$
\mathcal{L}=-\frac{\lambda^{2}}{4}\left(\partial_{\mu} \vec{\phi} \times \partial_{\nu} \vec{\phi}\right)^{2}-V
$$

The sigma-model and Skyrme terms are invariants under the global $S O(3)$ symmetry, whereas the potential breaks partially it, preserving only the subgroup $U(1)$ of the target space. The existence of such an unbroken subgroup $U(1)$ allows us to implement a local gauge symmetry by means of the introduction of a $U(1)$ gauge field of which the dynamics can be governed by the Maxwell action [28] or the Chern-Simons action [41,42] or both [43].

In the remainder of this section, we consider a restricted baby Skyrme model gauged solely with the Chern-Simons term described by the following Lagrangian density,

$$
\mathcal{L}=-\frac{\kappa}{4} \epsilon^{\sigma \mu \nu} A_{\sigma} F_{\mu \nu}-\frac{\lambda^{2}}{4}\left(D_{\mu} \vec{\phi} \times D_{\nu} \vec{\phi}\right)^{2}-V\left(\phi_{n}\right),
$$

where $\kappa$ is the Chern-Simons coupling constant, $A_{\mu}$ is the Abelian gauge field, and $F_{\mu \nu}=\partial_{\mu} A_{\nu}-\partial_{\nu} A_{\mu}$ is the field 
strength tensor. The minimal covariant derivative of the Skyrme field $D_{\mu} \vec{\phi}$ is given by

$$
D_{\mu} \vec{\phi}=\partial_{\mu} \vec{\phi}+g A_{\mu} \hat{n} \times \vec{\phi}
$$

where $g$ is the electromagnetic coupling constant. Here, we consider the gauge field with mass dimension 1 and the Skyrme field to be dimensionless. Hence, both the ChernSimons coupling constant $\kappa$ and the electromagnetic one $g$ become dimensionless, and the $\lambda$ coupling constant has mass dimension $-1 / 2$.

The gauge field equation obtained from of the Lagrangian density (3) is

$$
-\frac{\kappa}{2} \epsilon^{\mu \alpha \beta} F_{\alpha \beta}=J^{\mu},
$$

where $J^{\mu}$ is the conserved gauge current density,

$$
J^{\mu}=\lambda^{2} g\left[\vec{\phi} \cdot\left(D^{\mu} \vec{\phi} \times D^{\nu} \vec{\phi}\right)\right]\left(\partial_{\nu} \phi_{n}\right),
$$

with $\partial_{\nu} \phi_{n}=\hat{n} \cdot \partial_{\nu} \vec{\phi}$. Similarly, the equation of motion of the Skyrme field is

$\lambda^{2} D_{\mu}\left\{\left[\vec{\phi} \cdot\left(D^{\mu} \vec{\phi} \times D^{\beta} \vec{\phi}\right)\right] D_{\beta} \vec{\phi}\right\}+(\hat{n} \times \vec{\phi}) \frac{\partial V}{\partial \phi_{n}}=0$.

We are interested in time-independent solutions of the model; thus, we write down the respective equations of motion. The stationary Gauss law reads

$$
\kappa B=\lambda^{2} g^{2} A_{0}\left(\partial_{j} \phi_{n}\right)^{2},
$$

where $B=F_{12}=\epsilon_{i j} \partial_{i} A_{j}$ is the magnetic field. We now can express the scalar potential as

$$
A_{0}=\frac{\kappa}{\lambda^{2} g^{2}} \frac{B}{\left(\partial_{j} \phi_{n}\right)^{2}}
$$

Similarly, the Ampère law is given by

$$
\kappa \partial_{i} A_{0}=-\lambda^{2} g\left(\partial_{i} \phi_{n}\right) Q .
$$

By using (9), it is rewritten as

$$
\frac{\kappa^{2}}{\lambda^{2} g^{2}} \partial_{i}\left(\frac{B}{\left(\partial_{j} \phi_{n}\right)^{2}}\right)=-\lambda^{2} g\left(\partial_{i} \phi_{n}\right) Q,
$$

where we have introduced the quantity $Q$, defined by

$$
Q=\vec{\phi} \cdot\left(D_{1} \vec{\phi} \times D_{2} \vec{\phi}\right)
$$

The stationary equation of motion of the Skyrme field is

$$
\begin{aligned}
0= & (\hat{n} \times \vec{\phi}) \frac{\partial V}{\partial \phi_{n}}+\lambda^{2} \epsilon_{i j} D_{i}\left(Q D_{j} \vec{\phi}\right) \\
& +\lambda^{2} g^{2}(\hat{n} \times \vec{\phi}) \partial_{j}\left[\left(A_{0}\right)^{2}\left(\partial_{j} \phi_{n}\right)\right] .
\end{aligned}
$$

\section{A. BPS formalism: The frustrated implementation}

In the stationary regime, the energy density corresponding to the model (3) reads

$$
\varepsilon=\frac{\lambda^{2} g^{2}}{2}\left(A_{0}\right)^{2}\left(\partial_{j} \phi_{n}\right)^{2}+\frac{\lambda^{2}}{2} Q^{2}+V
$$

where we have used the identity

$$
\left(D_{i} \vec{\phi} \times D_{j} \vec{\phi}\right)^{2}=2 Q^{2}
$$

We first use the Gauss law (8), to express $A_{0}$ in terms of the magnetic field, such that the energy density (14) becomes

$$
\varepsilon=\frac{\kappa^{2}}{2 \lambda^{2} g^{2}} \frac{B^{2}}{\left(\partial_{j} \phi_{n}\right)^{2}}+\frac{\lambda^{2}}{2} Q^{2}+V,
$$

while the system energy is written as

$$
E=\int d^{2} \mathbf{x}\left\{\frac{\kappa^{2}}{2 \lambda^{2} g^{2}} \frac{B^{2}}{\left(\partial_{j} \phi_{n}\right)^{2}}+\frac{\lambda^{2}}{2} Q^{2}+V\right\} .
$$

After some algebraic manipulations, the implementation of the BPS formalism allows us to express the energy as

$$
\begin{aligned}
E= & \int d^{2} \mathbf{x}\left\{\frac{\lambda^{2}}{2}(Q \mp Z)^{2}+\frac{\kappa^{2}}{2 \lambda^{2} g^{2}} \frac{\left[B \pm\left(\partial_{j} \phi_{n}\right)^{2} W\right]^{2}}{\left(\partial_{j} \phi_{n}\right)^{2}}\right. \\
& \left. \pm \lambda^{2} Q Z \mp \frac{\kappa^{2} B W}{\lambda^{2} g^{2}}+V-\frac{\lambda^{2} Z^{2}}{2}-\frac{\kappa^{2}\left(\partial_{j} \phi_{n}\right)^{2} W^{2}}{2 \lambda^{2} g^{2}}\right\},
\end{aligned}
$$

where we have conveniently introduced two functions $W\left(\phi_{n}\right)$ and $Z\left(\phi_{n}\right)$ which should be determined a posteriori. Such a procedure is already utilized in the literature with the aim to attain a successful implementation of the BPS formalism. For example, it has already been used in the context of Skyrmions [28,42] and some generalized versions of the Maxwell-Higgs model [46].

By expressing the quantity $Q$ defined in (12) as

$$
Q=\vec{\phi} \cdot\left(\partial_{1} \vec{\phi} \times \partial_{2} \vec{\phi}\right)-g \epsilon_{i j} A_{j}\left(\partial_{i} \phi_{n}\right),
$$

the energy (18) becomes 


$$
\begin{aligned}
E= & \int d^{2} \mathbf{x}\left\{\frac{\kappa^{2}}{2 \lambda^{2} g^{2}} \frac{\left[B \pm\left(\partial_{j} \phi_{n}\right)^{2} W\right]^{2}}{\left(\partial_{j} \phi_{n}\right)^{2}}+\frac{\lambda^{2}}{2}(Q \mp Z)^{2}\right. \\
& \pm \lambda^{2} Z \vec{\phi} \cdot\left(\partial_{1} \vec{\phi} \times \partial_{2} \vec{\phi}\right) \mp \lambda^{2} g \epsilon_{i j} A_{j} Z\left(\partial_{i} \phi_{n}\right) \\
& \left.\mp \frac{\kappa^{2} B W}{\lambda^{2} g^{2}}+\left[V-\frac{\lambda^{2} Z^{2}}{2}-\frac{\kappa^{2}\left(\partial_{j} \phi_{n}\right)^{2} W^{2}}{2 \lambda^{2} g^{2}}\right]\right\} .
\end{aligned}
$$

The term $\vec{\phi} \cdot\left(\partial_{1} \vec{\phi} \times \partial_{2} \vec{\phi}\right)$ in the second row is related to the topological degree (topological charge or winding number) of the Skyrme field, which is defined by

$$
\operatorname{deg}[\vec{\phi}]=-\frac{1}{4 \pi} \int d^{2} \mathbf{x} \vec{\phi} \cdot\left(\partial_{1} \vec{\phi} \times \partial_{2} \vec{\phi}\right)=k,
$$

where $k$ is a non-null integer. Consequently, the term $\pm \lambda^{2} Z \vec{\phi} \cdot\left(\partial_{1} \vec{\phi} \times \partial_{2} \vec{\phi}\right)$ will provide the BPS energy.

To continue the implementation of the BPS formalism, we perform the following procedures in Eq. (20). First, we transform the fourth and fifth terms in a total derivative by imposing the condition

$Z\left(\partial_{j} \phi_{n}\right)=\frac{\kappa^{2}}{\lambda^{4} g^{3}} \partial_{i} W \quad$ such that $\quad Z=\frac{\kappa^{2}}{\lambda^{4} g^{3}} \frac{\partial W}{\partial \phi_{n}}$.

Second, in the third row, setting the term in square brackets to be null leads to the potential

$$
V=\frac{\kappa^{4}}{2 \lambda^{6} g^{6}}\left(\frac{\partial W}{\partial \phi_{n}}\right)^{2}+\frac{\kappa^{2}}{2 \lambda^{2} g^{2}}\left(\partial_{j} \phi_{n}\right)^{2} W^{2},
$$

where we see the function $W\left(\phi_{n}\right)$ plays the role of a "superpotential" such as has been pointed out in the literature $[28,42]$. It follows that the energy becomes

$$
\begin{aligned}
E= & \int d^{2} \mathbf{x}\left\{\frac{\kappa^{2}}{2 \lambda^{2} g^{2}} \frac{\left[B \pm\left(\partial_{j} \phi_{n}\right)^{2} W\right]^{2}}{\left(\partial_{j} \phi_{n}\right)^{2}}\right. \\
& +\frac{\lambda^{2}}{2}\left[Q \mp \frac{\kappa^{2}}{\lambda^{4} g^{3}} \frac{\partial W}{\partial \phi_{n}}\right]^{2} \pm \frac{\kappa^{2}}{\lambda^{2} g^{2}} \epsilon_{i j} \partial_{j}\left(W A_{i}\right) \\
& \left. \pm \frac{\kappa^{2}}{\lambda^{2} g^{3}} \frac{\partial W}{\partial \phi_{n}} \vec{\phi} \cdot\left(\partial_{1} \vec{\phi} \times \partial_{2} \vec{\phi}\right)\right\} .
\end{aligned}
$$

The squared terms would be the BPS equations, while the third term being a total derivative would give a null contribution to the total energy if $\lim _{\phi_{n} \rightarrow 1} W\left(\phi_{n}\right)=$ $\lim _{|\mathbf{x}| \rightarrow \infty} W\left(\phi_{n}\right)=0$. The fourth term related to the topological charge of the Skyrme field would provide the BPS limit for the total energy.

Until now, the implementation of the BPS formalism looks successful; however, there is a contradiction with the hypothesis about the functional dependence of the potential shown in the Lagrangian density (3). This contradiction can observed in Eq. (23) where the BPS potential now has explicit dependence on both the Skyrme field and its derivative. Consequently, the stationary Euler-Lagrange equation (13) of the Skyrme fields is not recovered from such BPS equations.

Despite our first attempt to implement the BPS formalism has been unsuccessful, the potential (23) suggests a way to introduce new terms in the model (3) making it capable to engender BPS configurations. The modified model with such a property is introduced in the next section.

\section{BPS CHERN-SIMONS BABY SKYRME MODEL}

The previous procedure suggests that the existence of BPS configurations can be well established in a modified version of the model (3). Such a modification is not arbitrary; the guidelines to perform such a change are given by the derivative term of Eq. (23), which indicates that a term proportional to $\left(\hat{n} \cdot D_{\mu} \vec{\phi}\right)^{2} W^{2}$ must be introduced in the Lagrangian density (3). Thus, the new model capable of engendering BPS configurations is described by the Lagrangian density

$$
\begin{aligned}
\mathcal{L}= & -\frac{\kappa}{4} \epsilon^{\sigma \mu \nu} A_{\sigma} F_{\mu \nu}-\frac{\lambda^{2}}{4}\left(D_{\mu} \vec{\phi} \times D_{\nu} \vec{\phi}\right)^{2} \\
& +\frac{\kappa^{2}}{2 \lambda^{2} g^{2}}\left(\hat{n} \cdot D_{\mu} \vec{\phi}\right)^{2} W^{2}-\mathcal{U},
\end{aligned}
$$

where both the dimensionless function $W$ and the potential $\mathcal{U}$ depend only in the variable $\phi_{n}$. The third term modifies the dynamics of the component along the direction $\hat{n}$ of the Skyrme field. In this way, the last two terms partially break the $S O(3)$ symmetry preserving the $U(1)$ subgroup of this symmetry.

It is worthwhile to point out that the model proposed in (25) has a $\mathcal{N}=2$ supersymmetry (SUSY) extension such as is shown in Ref. [44]. In other words, the model (3), despite possessing a $\mathcal{N}=1$ SUSY, does not admit a BPS structure. However, such as explained in Ref. [44], the $\mathcal{N}=$ 2 SUSY extension possessing a BPS or self-dual structure only is possible through the inclusion of the term proportional to $\left(\hat{n} \cdot D_{\mu} \vec{\phi}\right)^{2} W^{2}$. Specifically, the function $W$ is related to the target space area density and the Kähler metric of the sigma-model part [see Eq. (27) below].

The term $\left(\hat{n} \cdot D_{\mu} \vec{\phi}\right)^{2}$ in (25) can be expressed in the form

$$
\left(\hat{n} \cdot D_{\mu} \vec{\phi}\right)^{2}=D_{\mu} \vec{\phi} \cdot D^{\mu} \vec{\phi}-\left(\hat{n} \times D_{\mu} \vec{\phi}\right)^{2}
$$

allowing us to express the Lagrangian density (25) as

$$
\begin{aligned}
\mathcal{L}= & -\frac{\kappa}{4} \epsilon^{\sigma \mu \nu} A_{\sigma} F_{\mu \nu}+\frac{\kappa^{2}}{2 \lambda^{2} g^{2}} W^{2} D_{\mu} \vec{\phi} \cdot D^{\mu} \vec{\phi} \\
& -\frac{\lambda^{2}}{4}\left(D_{\mu} \vec{\phi} \times D_{\nu} \vec{\phi}\right)^{2}-\frac{\kappa^{2}}{2 \lambda^{2} g^{2}}\left(\hat{n} \times D_{\mu} \vec{\phi}\right)^{2} W^{2}-\mathcal{U} .
\end{aligned}
$$


The second term is a generalized gauged sigma model with the function $W$ playing the role of the generalizing function, and the third one is the gauged Skyrme term. In other words, the new model (25) is a type of generalized Chern-Simons baby Skyrme model modified by the term proportional to $\left(\hat{n} \times D_{\mu} \vec{\phi}\right)^{2} W^{2}$.

We point out that the gauge field equation coming from the Lagrangian density (25) is exactly the same given by Eq. (5); i.e., the introduction of the function $W\left(\phi_{n}\right)$ does not modify the gauge field equation of motion.

The equation of motion of the Skyrme field obtained from the Lagrangian density (25) is

$$
\begin{aligned}
0= & \lambda^{2} D_{\mu}\left\{\left(D_{\beta} \vec{\phi}\right)\left[\vec{\phi} \cdot\left(D^{\mu} \vec{\phi} \times D^{\beta} \vec{\phi}\right)\right]\right\} \\
& +(\hat{n} \times \vec{\phi})\left\{\frac{\partial \mathcal{U}}{\partial \phi_{n}}+\frac{\kappa^{2}}{\lambda^{2} g^{2}} \partial_{\mu}\left[\left(\hat{n} \cdot \partial^{\mu} \vec{\phi}\right) W^{2}\right]\right. \\
& \left.-\frac{\kappa^{2}}{2 \lambda^{2} g^{2}}\left(\hat{n} \cdot \partial_{\nu} \vec{\phi}\right)^{2} \frac{\partial W^{2}}{\partial \phi_{n}}\right\}
\end{aligned}
$$

the stationary version of which reads

$$
\begin{aligned}
& \lambda^{2} \epsilon_{i j} D_{i}\left(Q D_{j} \vec{\phi}\right) \\
& =-(\hat{n} \times \vec{\phi})\left\{\frac{\partial \mathcal{U}}{\partial \phi_{n}}+\frac{\kappa^{2}\left(\partial_{j} \phi_{n}\right)^{2}}{2 \lambda^{2} g^{2}} \frac{\partial W^{2}}{\partial \phi_{n}}\right. \\
& \left.\quad+\frac{\kappa^{2}}{\lambda^{2} g^{2}} \partial_{j}\left[\frac{B^{2}}{\left(\partial_{j} \phi_{n}\right)^{3}}\right]-\frac{\kappa^{2}}{\lambda^{2} g^{2}} \partial_{j}\left[\left(\partial_{j} \phi_{n}\right) W^{2}\right]\right\},
\end{aligned}
$$

where we have used (9) to replace the scalar potential with the magnetic field.

In the following, we are going to show how to build the BPS formalism determining the self-interacting potential $\mathcal{U}\left(\phi_{n}\right)$ which allows us to obtain a lower bound for the energy and the self-dual equations satisfied by the soliton configurations saturating a BPS bound.

\section{A. BPS configurations}

The stationary energy density is written as

$\varepsilon=\frac{\kappa^{2}}{2 \lambda^{2} g^{2}} \frac{B^{2}}{\left(\partial_{j} \phi_{n}\right)^{2}}+\frac{\kappa^{2}}{2 \lambda^{2} g^{2}}\left(\partial_{j} \phi_{n}\right)^{2} W^{2}+\frac{\lambda^{2}}{2} Q^{2}+\mathcal{U}$,

where we have used the Gauss law (8) to express the scalar potential in terms of the magnetic field.

We now implement the BPS formalism, which, after some algebraic manipulations, allows us to write the total energy as

$$
\begin{aligned}
E= & \int d^{2} \mathbf{x}\left\{\frac{\kappa^{2}}{2 \lambda^{2} g^{2}} \frac{\left[B \pm\left(\partial_{j} \phi_{n}\right)^{2} W\right]^{2}}{\left(\partial_{j} \phi_{n}\right)^{2}}\right. \\
& +\frac{\lambda^{2}}{2}\left[Q \mp \frac{\sqrt{2 \mathcal{U}}}{\lambda}\right]^{2} \pm \lambda \sqrt{2 \mathcal{U}} \vec{\phi} \cdot\left(\partial_{1} \vec{\phi} \times \partial_{2} \vec{\phi}\right) \\
& \left.\mp \lambda g \epsilon_{i j} A_{j}\left(\partial_{i} \phi_{n}\right) \sqrt{2 \mathcal{U}} \mp \frac{\kappa^{2} B W}{\lambda^{2} g^{2}}\right\} .
\end{aligned}
$$

The third term is related to the topological charge of the Skyrme field, and as we will see below, it provides the Bogomol'nyi limit for the total energy. The implementation of the BPS formalism is finished by transforming the last two terms in a total derivative by setting

$$
\left(\partial_{i} \phi_{n}\right) \sqrt{2 \mathcal{U}}=\frac{\kappa^{2}}{\lambda^{3} g^{3}} \partial_{i} W
$$

Consequently, the self-interacting potential able to engender self-dual configurations becomes

$$
\mathcal{U}\left(\phi_{n}\right)=\frac{\kappa^{4}}{2 \lambda^{6} g^{6}}\left(\frac{\partial W}{\partial \phi_{n}}\right)^{2} .
$$

This clearly shows the role of a superpotential fulfilled by the function $W\left(\phi_{n}\right)$ in the model (25). The superpotential $W\left(\phi_{n}\right)$ must be constructed or proposed in order for the potential to satisfy the vacuum condition

$$
\lim _{\phi_{n} \rightarrow 1} \mathcal{U}\left(\phi_{n}\right)=\lim _{|\mathbf{x}| \rightarrow \infty} \mathcal{U}\left(\phi_{n}\right)=0,
$$

implying the following boundary condition on the superpotential:

$$
\lim _{\phi_{n} \rightarrow 1} \frac{\partial W}{\partial \phi_{n}}=\lim _{|\mathbf{x}| \rightarrow \infty} \frac{\partial W}{\partial \phi_{n}}=0 .
$$

Since the BPS potential was fixed, the energy (31) reads

$$
\begin{aligned}
E= & \int d^{2} \mathbf{x}\left\{\frac{\kappa^{2}}{2 \lambda^{2} g^{2}} \frac{\left[B \pm\left(\partial_{j} \phi_{n}\right)^{2} W\right]^{2}}{\left(\partial_{j} \phi_{n}\right)^{2}}\right. \\
& +\frac{\lambda^{2}}{2}\left[Q \mp \frac{\kappa^{2}}{\lambda^{4} g^{3}} \frac{\partial W}{\partial \phi_{n}}\right]^{2} \\
& \left. \pm \frac{\kappa^{2}}{\lambda^{2} g^{3}} \frac{\partial W}{\partial \phi_{n}} \vec{\phi} \cdot\left(\partial_{1} \vec{\phi} \times \partial_{2} \vec{\phi}\right) \mp \frac{\kappa^{2}}{\lambda^{2} g^{2}} \epsilon_{i j} \partial_{i}\left(W A_{j}\right)\right\} .
\end{aligned}
$$

The contribution of the total derivative to the total energy becomes null, i.e.,

$$
\int d^{2} \mathbf{x} \varepsilon_{i j} \partial_{i}\left(A_{j} W\right)=0
$$

by considering the superpotential satisfying the following boundary condition: 


$$
\lim _{\phi_{n} \rightarrow 1} W\left(\phi_{n}\right)=\lim _{|\mathbf{x}| \rightarrow \infty} W\left(\phi_{n}\right)=0 .
$$

Hence, we now write the total energy as

$$
E=E_{\mathrm{BPS}}+\check{E},
$$

with $E_{\mathrm{BPS}}$ defining the energy lower bound

$$
E_{\mathrm{BPS}}= \pm \frac{\kappa^{2}}{\lambda^{2} g^{3}} \int d^{2} \mathbf{x} \vec{\phi} \cdot\left(\partial_{1} \vec{\phi} \times \partial_{2} \vec{\phi}\right) \frac{\partial W}{\partial \phi_{n}}
$$

and $\check{E}$ given by

$$
\begin{aligned}
\check{E}= & \int d^{2} \mathbf{x}\left\{\frac{\kappa^{2}}{2 \lambda^{2} g^{2}} \frac{\left[B \pm\left(\partial_{j} \phi_{n}\right)^{2} W\right]^{2}}{\left(\partial_{j} \phi_{n}\right)^{2}}\right. \\
& \left.+\frac{\lambda^{2}}{2}\left(Q \mp \frac{\kappa^{2}}{\lambda^{4} g^{3}} \frac{\partial W}{\partial \phi_{n}}\right)^{2}\right\} .
\end{aligned}
$$

From the expression of the total energy (39), we observe that the following inequality is always satisfied:

$$
E \geq E_{\mathrm{BPS}} .
$$

The lower bound is saturated, i.e., $\breve{E}=0$, if the fields satisfy the self-dual or BPS equations

$$
\begin{aligned}
& B=\mp\left(\partial_{j} \phi_{n}\right)^{2} W, \\
& Q= \pm \frac{\kappa^{2}}{\lambda^{4} g^{3}} \frac{\partial W}{\partial \phi_{n}} .
\end{aligned}
$$

These BPS configurations can be considered as classical solutions related to an extended supersymmetric theory $[47,48]$ of the model $(25)$. Indeed, such affirmation was shown in Ref. [44]; i.e., the BPS equations belong to a $\mathcal{N}=2$ SUSY extension model of which the bosonic sector would be given by the Lagrangian (25). Besides, the solutions of Eqs. (43) and (44) are of the type 1/4-BPS, corresponding to the nontrivial phase of the $\mathcal{N}=2$ SUSY extension model.

\section{B. Equivalence between the BPS and Euler-Lagrange equations}

In this section, we will show that the BPS equations (43)-(44) allows us recover the Ampere law (11) and the Skyrme field equation (29) provided by the Lagrangian density (25).

\section{Ampre's law}

To recover the Ampère law (11), we employ the first BPS (43)

$$
\partial_{i}\left(\frac{B}{\left(\partial_{j} \phi_{n}\right)^{2}}\right)=\mp \partial_{i} W=\mp \frac{\partial W}{\partial \phi_{n}} \partial_{i} \phi_{n} .
$$

We now use the second BPS (44) to substitute $\frac{\partial W}{\partial \phi_{n}}$ above; thus, we attain our objective,

$$
\partial_{i}\left(\frac{B}{\left(\partial_{j} \phi_{n}\right)^{2}}\right)=-\frac{\lambda^{4} g^{3}}{\kappa^{2}}\left(\partial_{i} \phi_{n}\right) Q .
$$

\section{Skyrmion field equation}

With the aim of recovering the Skyrmion field equation in the BPS limit, we begin with the second BPS (44) by doing

$$
\begin{aligned}
\lambda^{2} \epsilon_{i j} D_{i}\left(Q D_{j} \vec{\phi}\right)= & \pm \frac{\kappa^{2}}{\lambda^{2} g^{3}} \epsilon_{i j} D_{i}\left(\frac{\partial W}{\partial \phi_{n}} D_{j} \vec{\phi}\right) \\
= & \pm \frac{\kappa^{2}}{\lambda^{2} g^{3}} \frac{\partial^{2} W}{\partial \phi_{n}^{2}} \epsilon_{i j}\left(\partial_{i} \phi_{n}\right) D_{j} \vec{\phi} \\
& \pm \frac{\kappa^{2}}{2 \lambda^{2} g^{3}} \frac{\partial W}{\partial \phi_{n}} \epsilon_{i j}\left[D_{i}, D_{j}\right] \vec{\phi}
\end{aligned}
$$

By using the identities

$$
\begin{gathered}
\epsilon_{i j}\left(\partial_{i} \phi_{n}\right) D_{j} \vec{\phi}=-Q(\hat{n} \times \vec{\phi}), \\
\epsilon_{i j}\left[D_{i}, D_{j}\right] \vec{\phi}=2 g B(\hat{n} \times \vec{\phi}),
\end{gathered}
$$

we obtain

$$
\begin{aligned}
\lambda^{2} \epsilon_{i j} D_{i}\left(Q D_{j} \vec{\phi}\right)= & \mp \frac{\kappa^{2}}{\lambda^{2} g^{3}} \frac{\partial^{2} W}{\partial \phi_{n}^{2}} Q(\hat{n} \times \vec{\phi}) \\
& \pm \frac{\kappa^{2}}{\lambda^{2} g^{2}} \frac{\partial W}{\partial \phi_{n}} B(\hat{n} \times \vec{\phi}) .
\end{aligned}
$$

We now substitute $B$ and $Q$ with the expressions provided by the BPS equations, Eqs. (43) and (44), respectively, to get

$$
\begin{aligned}
\lambda^{2} \epsilon_{i j} D_{i}\left(Q D_{j} \vec{\phi}\right)= & -\frac{\kappa^{4}}{2 \lambda^{6} g^{6}} \frac{\partial}{\partial \phi_{n}}\left(\frac{\partial W}{\partial \phi_{n}}\right)^{2}(\hat{n} \times \vec{\phi}) \\
& -\frac{\kappa^{2}}{2 \lambda^{2} g^{2}}\left(\partial_{j} \phi_{n}\right)^{2} \frac{\partial W^{2}}{\partial \phi_{n}}(\hat{n} \times \vec{\phi}) .
\end{aligned}
$$

Finally, we use Eq. (33) to write

$$
\begin{aligned}
0= & \lambda^{2} \epsilon_{i j} D_{i}\left(Q D_{j} \vec{\phi}\right) \\
& +(\hat{n} \times \vec{\phi})\left\{\frac{\partial \mathcal{U}}{\partial \phi_{n}}+\frac{\kappa^{2}}{2 \lambda^{2} g^{2}}\left(\partial_{j} \phi_{n}\right)^{2} \frac{\partial W^{2}}{\partial \phi_{n}}\right\},
\end{aligned}
$$

which is the Skyrmion field equation (29) in the BPS limit. 
Therefore, we have verified that the BPS equations recover the Euler-Lagrange equations of the model (25) with the potential given by (33).

\section{ROTATIONALLY SYMMETRIC BPS SKYRMIONS}

Without loss of generality, we set $\hat{n} \equiv \hat{n}_{3}=(0,0,1)$, such that $\hat{n} \cdot \vec{\phi}=\phi_{n}=\phi_{3}$, and consider the ansatz for the Skyrme field,

$$
\left(\begin{array}{l}
\phi_{1} \\
\phi_{2} \\
\phi_{3}
\end{array}\right)=\left(\begin{array}{c}
\sin f(r) \cos N \theta \\
\sin f(r) \sin N \theta \\
\cos f(r)
\end{array}\right),
$$

where $N=\operatorname{deg}[\vec{\phi}]$ is the winding number of the Skyrme field. For the gauge field, we use

$$
A_{k}=-\varepsilon_{k j} \frac{x_{j}}{g r^{2}}[a(r)-N]
$$

thus, the magnetic field is given by

$$
B=\frac{1}{g r} \frac{d a}{d r} .
$$

The functions $f(r)$ and $a(r)$ are well behaved and must satisfy the boundary conditions,

$$
\begin{gathered}
f(0)=\pi, \quad f(\infty)=0, \\
a(0)=N, \quad a(\infty)=a_{\infty},
\end{gathered}
$$

where $a_{\infty}$ is a finite quantity.

To perform our analysis, we introduce the field redefinition

$$
h=\frac{1}{2}\left(1+\phi_{3}\right)=\frac{1}{2}(1+\cos f),
$$

with the field $h(r)$ satisfying the boundary conditions

$$
h(0)=0, \quad h(\infty)=1 .
$$

We consider superpotentials $W$, satisfying the following boundary conditions at origin

$$
\lim _{r \rightarrow 0} W(h)=W_{0}, \quad \lim _{r \rightarrow 0} \frac{d W}{d r}=c t e
$$

( $W_{0}$ being a finite quantity). From Eqs. (35) and (38), we obtain the boundary conditions for $r \rightarrow \infty$,

$$
\lim _{r \rightarrow \infty} W(h)=0, \quad \lim _{r \rightarrow \infty} \frac{d W}{d r}=0,
$$

the last one guaranteeing the superpotential is able to generate a potential satisfying the vacuum condition,

$$
\mathcal{U}(\infty) \equiv \mathcal{U}(h=1)=0 .
$$

Under the ansatz, the BPS equations become

$$
\begin{gathered}
\frac{1}{r} \frac{d a}{d r}=\mp 4 g\left(\frac{d h}{d r}\right)^{2} W, \\
\frac{a}{r} \frac{d h}{d r}=\mp \frac{\kappa^{2}}{4 \lambda^{4} g^{3}} \frac{\partial W}{\partial h} .
\end{gathered}
$$

Similarly, the BPS energy density reads

$$
\varepsilon_{\mathrm{BPS}}=\frac{4 \kappa^{2}}{\lambda^{2} g^{2}} W^{2}\left(\frac{d h}{d r}\right)^{2}+\frac{\kappa^{4}}{4 \lambda^{6} g^{6}}\left(\frac{\partial W}{\partial h}\right)^{2},
$$

while the BPS energy (40) becomes

$$
\mathcal{E}_{\mathrm{BPS}}= \pm 2 \pi N \frac{\kappa^{2}}{\lambda^{2} g^{3}} W(0) .
$$

The total magnetic flux $\Phi$ is computed to be

$$
\Phi=2 \pi \int_{0}^{\infty} r d r B=\frac{2 \pi}{g}\left[a_{\infty}-N\right],
$$

which is, in general, a nonquantized quantity.

By integrating the Gauss law (8), we also obtain the total electric charge $\mathcal{Q}_{\mathrm{em}}$ being proportional to the total magnetic flux $\Phi$,

$$
\mathcal{Q}_{\mathrm{em}}=\frac{\kappa}{g} \Phi,
$$

where the total electric charge was defined by

$$
\mathcal{Q}_{\mathrm{em}}=g \lambda^{2} \int d^{2} \mathbf{x} A_{0}\left(\hat{n} \cdot \partial_{j} \vec{\phi}\right)^{2} .
$$

In Sec. V, the numerical analysis has shown, for sufficiently large values of $g$, that the magnetic flux becomes almost a topologically quantized observable. This effective quantization implies the total electric charge is also quantized.

\section{A. Behavior of the profiles at origin}

We first solve the BPS equations (63) and (64) around $r=0$ by considering the boundary conditions

$$
h(0)=0, \quad a(0)=N, \quad W(0)=W_{0} .
$$

The superpotential $W(h)$ is considered to be a well-behaved function such that behavior for the field profiles $h(r)$ and $a(r)$ results in 


$$
\begin{aligned}
& h(r) \approx-\frac{\kappa^{2}\left(W_{h}\right)_{h=0}}{8 N \lambda^{4} g^{3}} r^{2}+\frac{\kappa^{4}\left(W_{h}\right)_{h=0}\left(W_{h h}\right)_{h=0}}{128 N^{2} \lambda^{8} g^{6}} r^{4}, \\
& a(r) \approx N-\frac{\kappa^{4} W_{0}\left(W_{h}\right)_{h=0}^{2}}{16 N^{2} \lambda^{8} g^{5}} r^{4}+\frac{\kappa^{6} W_{0}\left(W_{h}\right)_{h=0}^{2}\left(W_{h h}\right)_{h=0}}{96 N^{3} \lambda^{12} g^{8}} r^{6},
\end{aligned}
$$

where $W_{h}$ and $W_{h h}$ represent the first and second derivatives of $W(h)$ with respect to $h$, respectively.

The magnetic field behavior near the origin is

$|B(r)| \approx \frac{\kappa^{4} W_{0}\left(W_{h}\right)_{h=0}^{2}}{4 N^{2} \lambda^{8} g^{6}} r^{2}-\frac{\kappa^{6} W_{0}\left(W_{h}\right)_{h=0}^{2}\left(W_{h h}\right)_{h=0}}{16 N^{3} \lambda^{12} g^{9}} r^{4}$,

and the BPS energy density behaves as

$$
\begin{aligned}
\varepsilon_{\mathrm{BPS}}(r) \approx & \frac{\kappa^{4}\left(W_{h}\right)_{h=0}^{2}}{4 \lambda^{6} g^{6}} \\
& +\frac{\kappa^{6}\left(W_{0}\right)^{2}\left(W_{h}\right)_{h=0}^{2}}{4 N^{2} \lambda^{10} g^{9}}\left[g-\frac{N\left(W_{h h}\right)_{h=0}}{4\left(W_{0}\right)^{2}}\right] r^{2} .
\end{aligned}
$$

For small values of $g$, the BPS energy density has greater amplitudes.

\section{B. Behavior of the profiles for large values of $\boldsymbol{r}$}

The analysis for sufficiently large values of $r$ is performed by considering the boundary conditions

$$
h(R)=1, \quad a(R)=a_{R}, \quad W(R)=0,
$$

where $R>0$ and $a_{R}$ is a real number. For a finite $R$, it defines a maximum radius (size) of the topological defect characterizing the soliton so-called compacton. On the other hand, when $R \rightarrow \infty$ there is topological defects whose tail decays follows an exponential law or a power law.

We have considered that the superpotential $W(h)$ behaves when $r \rightarrow R$ as

$$
W(h) \approx(1-h)^{\sigma},
$$

with the parameter $\sigma>1$. Until now, we have found three types of soliton solutions:

(i) For $1<\sigma<2$, there are compacton solitons.

(ii) For $\sigma=2$, the soliton tail decays, following a exponential law of type $e^{-\alpha r^{2}}, \alpha>0$.

(iii) For $\sigma>2$, the solitons have a power-law decay of type $r^{-\beta}, \beta>0$.

\section{Behavior of the profiles for $1<\sigma<2$}

We consider that the compacton has a maximum radius $R$ and the superpotential behaves at $r=R$ as

$$
W(h) \approx W_{R}(1-h)^{\sigma} .
$$

By considering it and solving the BPS equations, we find the profile functions behave as

$$
\begin{aligned}
& h(r) \approx 1-\left(C_{R}^{(h)}\right)^{1 /(2-\sigma)}(R-r)^{1 /(2-\sigma)}, \\
& a(r) \approx a_{R}+C_{R}^{(a)}(R-r)^{2 \sigma /(2-\sigma)},
\end{aligned}
$$

where

$$
\begin{aligned}
C_{R}^{(h)} & =\frac{\sigma(2-\sigma) \kappa^{2} R W_{R}}{4 \lambda^{4} g^{3} a_{R}}, \\
C_{R}^{(a)} & =\frac{2 g R W_{R}}{\sigma(2-\sigma)}\left(C_{R}^{(h)}\right)^{(2+\sigma) /(2-\sigma)} .
\end{aligned}
$$

\section{Behavior of the profiles for $\sigma=2$}

For the superpotentials following the asymptotic behavior

$$
W(h) \approx W_{\infty}^{(2)}(1-h)^{2},
$$

when $r \rightarrow \infty$, the prole functions behave

$$
\begin{aligned}
& h(r) \approx 1-C e^{-M^{2} r^{2}}, \\
& a(r) \approx a_{\infty}+2 g W_{\infty}^{(2)} C^{4} M^{2} r^{2} e^{-4 M^{2} r^{2}},
\end{aligned}
$$

where the quantity $M$ is given by

$$
M^{2}=\frac{\kappa^{2} W_{\infty}^{(2)}}{4 \lambda^{4} g^{3} a_{\infty}} .
$$

This verifies that the soliton tail has an exponential decay law.

\section{Behavior of the profiles for $\sigma>2$}

We consider that the superpotential for $r \rightarrow \infty$ behaves as

$$
W(h) \approx W_{\infty}(1-h)^{\sigma},
$$

and the profiles have the behavior

$$
\begin{aligned}
& h(r) \approx 1-\left(\frac{C^{(\infty)}}{r^{2}}\right)^{1 /(\sigma-2)}, \\
& a(r) \approx a_{\infty}+\frac{8 g W_{\infty}}{\left(\sigma^{2}-4\right)}\left(\frac{C^{(\infty)}}{r^{2}}\right)^{(2+\sigma) /(\sigma-2)},
\end{aligned}
$$

where

$$
C^{(\infty)}=\frac{8 \lambda^{4} g^{3} a_{\infty}}{\kappa^{2} W_{\infty} \sigma(\sigma-2)} .
$$


We see that the profiles follow a power-law decay but the gauge field decays faster than the Skyrme field.

\section{NUMERICAL SOLUTION OF THE BPS EQUATIONS}

\section{A. Compacton solutions}

We have solved the BPS equations (63) and (64) for the superpotential

$$
W(h)=W_{0}(1-h)^{3 / 2},
$$

which provides the potential

$$
\mathcal{U}(h)=\frac{9 \kappa^{4} W_{0}^{2}}{32 \lambda^{6} g^{6}}(1-h) .
$$

This is equivalent to the well-known "old baby Skyrme potential" [28].

In our first analysis, we solved the BPS equations (63) and (64) by fixing $N=1, \kappa=1, W_{0}=1$, and $\lambda=2.5$ and running the electromagnetic coupling constant $g$. The compacton solutions are depicted in Figs. 1-4.

The Skyrme field profile $h(r)$ is depicted in Fig. 1 for various values of $g$. The colored solid lines represent the profiles of the $h(r)$ in the interval $0 \leq r \leq R$, and the respective colored pointed lines represent the vacuum value, $h=1$, in the interval $R \leq r<\infty$. The compacton radius $R$ for various values of $g$ is shown in Fig. 5.

Figure 2 depicts the gauge field profile $a(r)$. Similar to the description given in Fig. 1, the colored solid lines represent the profiles of the $a(r)$ in the interval $0 \leq r \leq R$, and the respective colored pointed lines represent the vacuum value, $a(R)=a_{R}$, in the interval $R \leq r<\infty$. The profiles show that the vacuum value, $a(R)=a_{R}$, diminishes whenever $g$ increases.

The profiles of the magnetic field are presented in Fig. 3. They are ringlike structures of which the maximum for

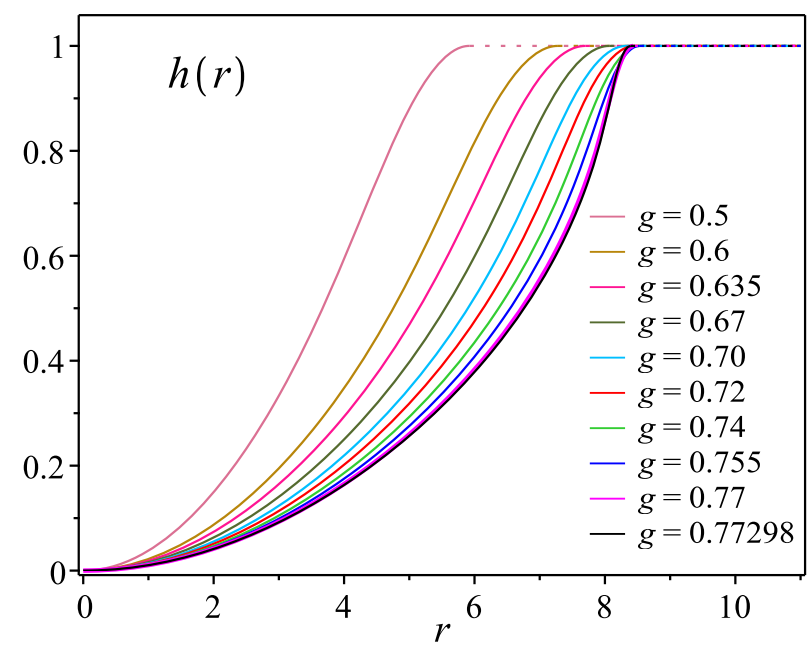

FIG. 1. The Skyrme field profile $h(r)$.

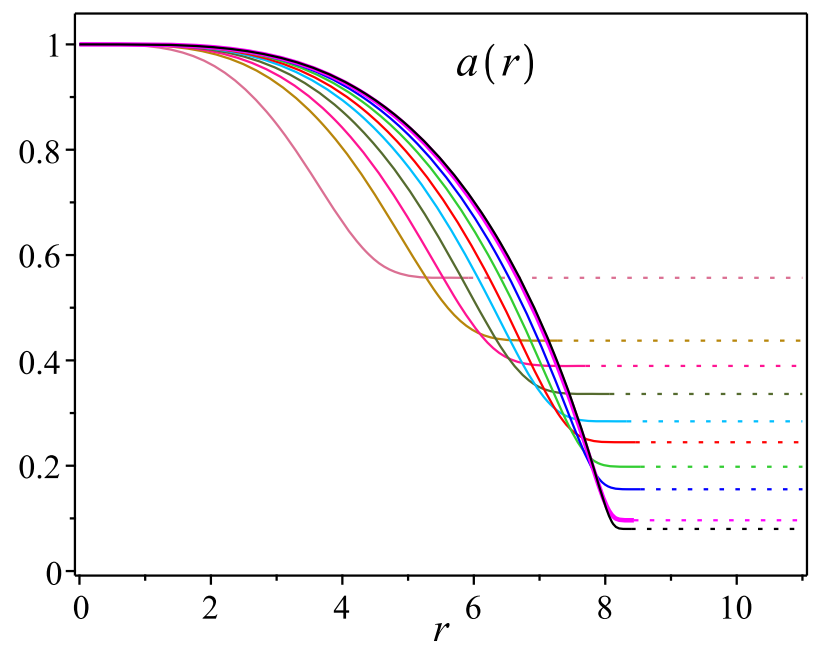

FIG. 2. The gauge field profile $a(r)$.

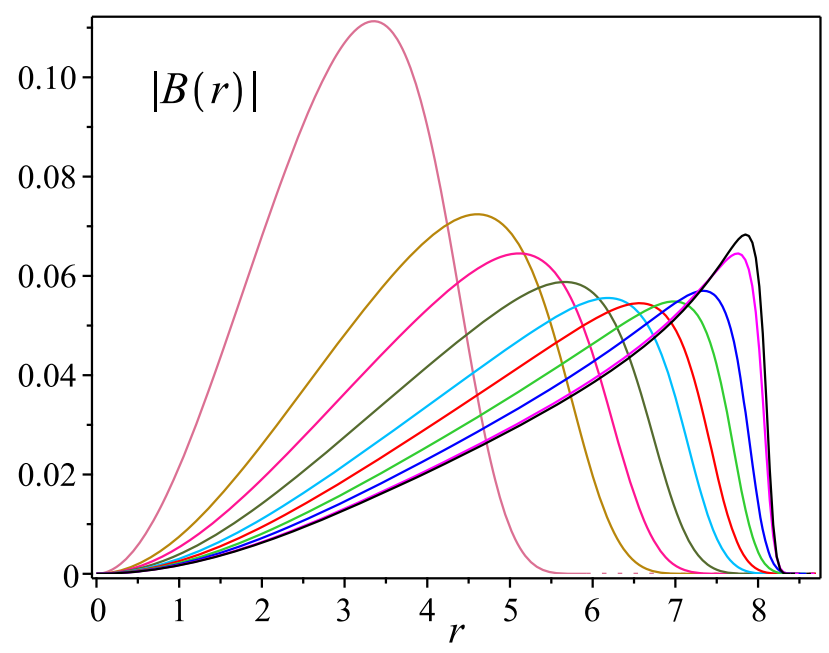

FIG. 3. The magnetic field $B(r)$.

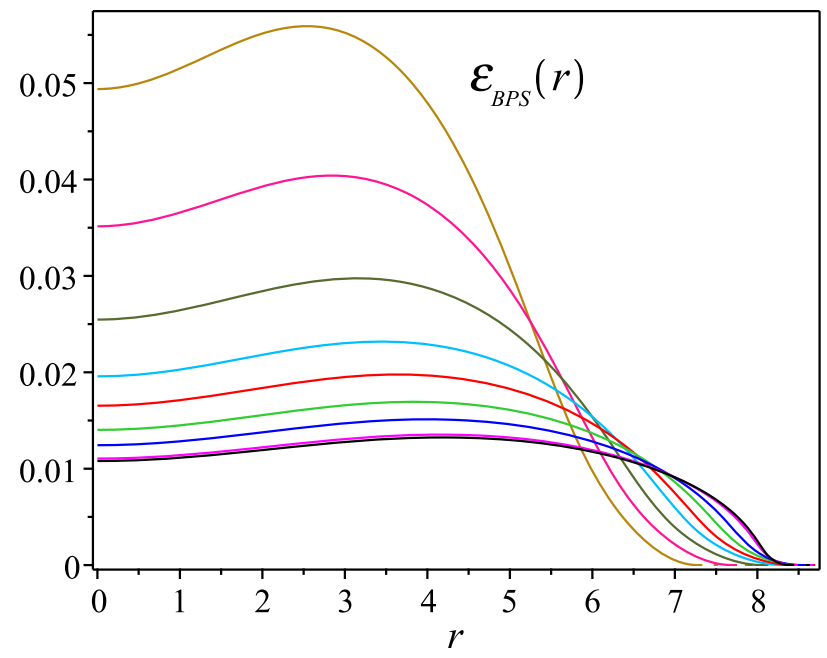

FIG. 4. The BPS energy density $\varepsilon_{\mathrm{BPS}}(r)$. 


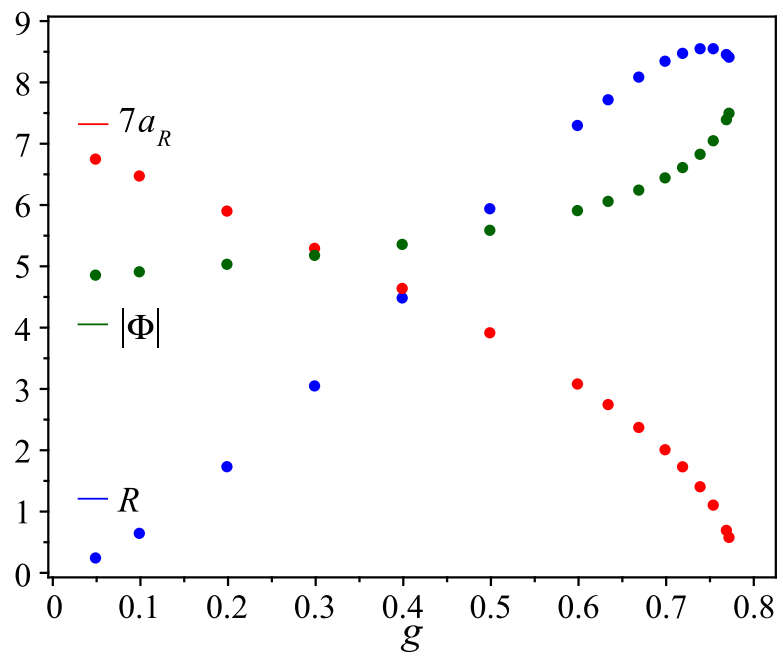

FIG. 5. Behaviors of the compacton radius $R$ vs $g$ (blue color), the gauge vacuum value $a_{R}$ vs $g$ (red color), and the magnetic flux $|\Phi|$ vs $g$ (green color) of compacton solutions of the BPS equations (63) and (64).

small $g$ is located close to the origin, whereas for sufficiently larger values of $g$, the maximum moves its position to very near the frontier of the compacton. The amplitude of the maximum value of the magnetic field is greater for small values of $g$. In our case, it happens for $0<g<0.5$; despite not being shown in the figure, it can be seen clearly from the behavior given by Eq. (73).

The profiles of the BPS energy density (65) are presented in Fig. 4. The behavior at the origin is given by [see Eq. (74)]

$\varepsilon_{\mathrm{BPS}} \approx \frac{23.04 \times 10^{-4}}{g^{6}}+\frac{5898.24 \times 10^{-8}}{g^{9}}[g-0.1875] r^{2}$.

For small values of $g$, the profiles possess a lumplike format at the origin. These profiles are not presented in the figure because their amplitudes (for $0<g<0.1875$ ) are bigger than the ones shown there. For sufficiently large values of $g$, the profiles acquire a ringlike form; in our case, Fig. 4 shows such structures for $g>0.6$.

The dependence of the compacton radius $R$ vs $g$, the gauge vacuum value $a_{R}$ vs $g$, and the total magnetic flux $|\Phi|$ vs $g$ (by fixing all other parameters) are shown in Fig. 5 for $N=1$, $\kappa=1, W_{0}=1$, and $\lambda=2.5$. We have observed that the vacuum value, $a(R)=a_{R}$, diminishes whenever $g$ increases, i.e., $a_{R} \rightarrow 0$ for sufficiently large values of $g$. Consequently, we get

$$
\Phi \rightarrow-\frac{2 \pi}{g} N, \quad \mathcal{Q}_{\mathrm{em}} \rightarrow-\frac{2 \pi \kappa}{g^{2}} N,
$$

being, therefore, quantities topologically quantized.

From Figs. 2 and 5, we observe clearly that the compacton radius $R$ possess a maximum value for some specific magnitude of the electromagnetic coupling

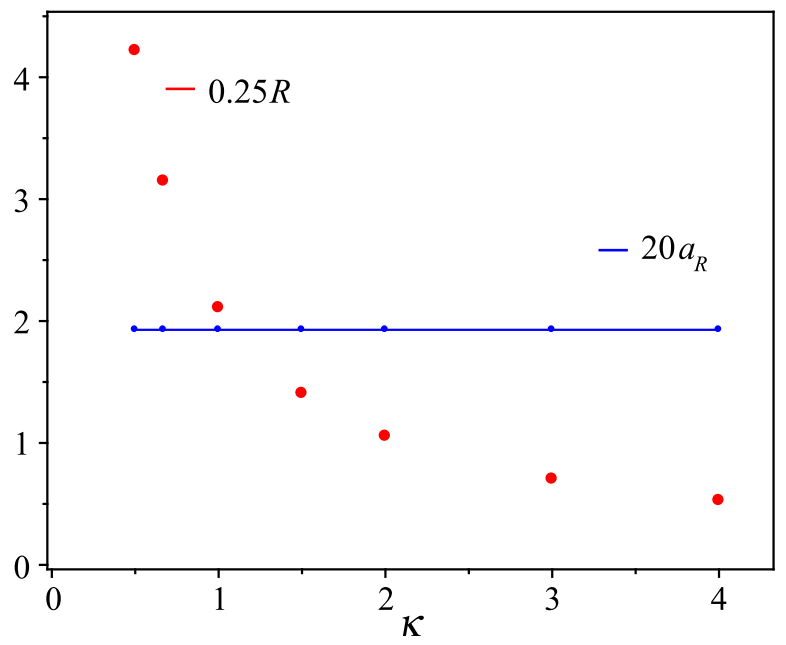

FIG. 6. Compacton radius $R$ vs $\kappa$ (red dots) and the gauge field vacuum value $a_{R}$ vs $\kappa$ (blue dotted line).

constant $g$. In both figures, $g$ is the only free parameter whereas all other are fixed.

Similarly, we have analyzed the dependence of the compacton radius $R$ vs $\kappa$ and the gauge vacuum value $a_{R}$ vs $\kappa$ (by fixing all other parameters). The numerical analysis has shown that the radius is inversely proportional to $\kappa\left(R \propto \kappa^{-1}\right)$, whereas the gauge vacuum value $a_{R}$ remains constant. Figure 6 shows $R$ vs $\kappa$ for $N=1$, $g=0.77, \quad W_{0}=1, \quad$ and $\lambda=2.5$, whereas $a_{R}=$ 0.0964097741 for all values of $\kappa$.

Our third analysis looks at the dependence of compacton radius $R$ vs $\lambda$ and the gauge vacuum value $a_{R}$ vs $\lambda$ (for all others parameters set). The numerical analysis shows that the radius depends quadratically with $\lambda\left(R \propto \lambda^{2}\right)$, whereas the gauge vacuum value $a_{R}$ remains constant. Such a dependence is depicted in Fig. 7 for $N=1, g=0.77$,

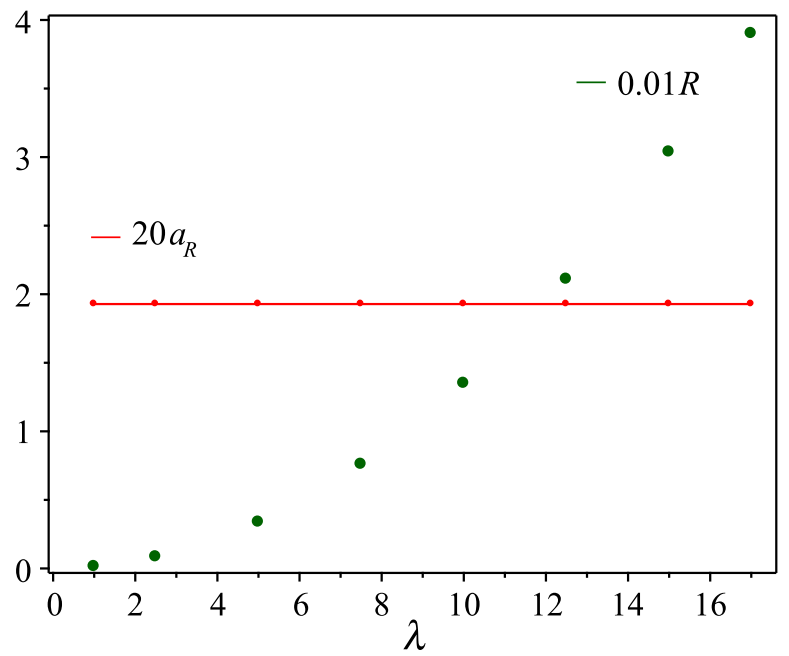

FIG. 7. Compacton radius $R$ vs $\lambda$ (green dots) and the gauge field vacuum value $a_{R}$ vs $\lambda$ (red dotted line). 


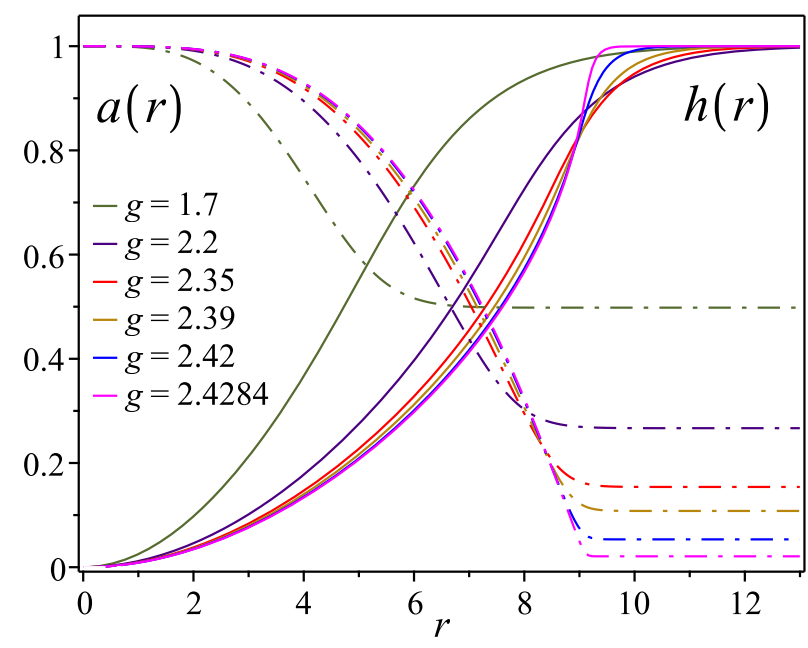

FIG. 8. Profiles of the Skyrme field $h(r)$ and the gauge field $a(r)$ with exponential decay engendered by the superpotential (94).

$W_{0}=1$, and $\kappa=1$, whereas $a_{R}=0.0964097741$ for all values of $\lambda$.

Until now, our analysis of the compacton solitons has allowed us to conclude that the gauge vacuum value $a_{R}$ only depends on the electromagnetic coupling constant $g$; consequently, the total magnetic flux is independent from the values of $\kappa$ and $\lambda$, and it becomes quantized for sufficiently large values of $g$.

\section{B. Solitons with exponential-law decay}

We have solved the BPS equations (63) and (64) for the superpotential

$$
W(h)=W_{0}(1-h)^{2},
$$

which provides the potential

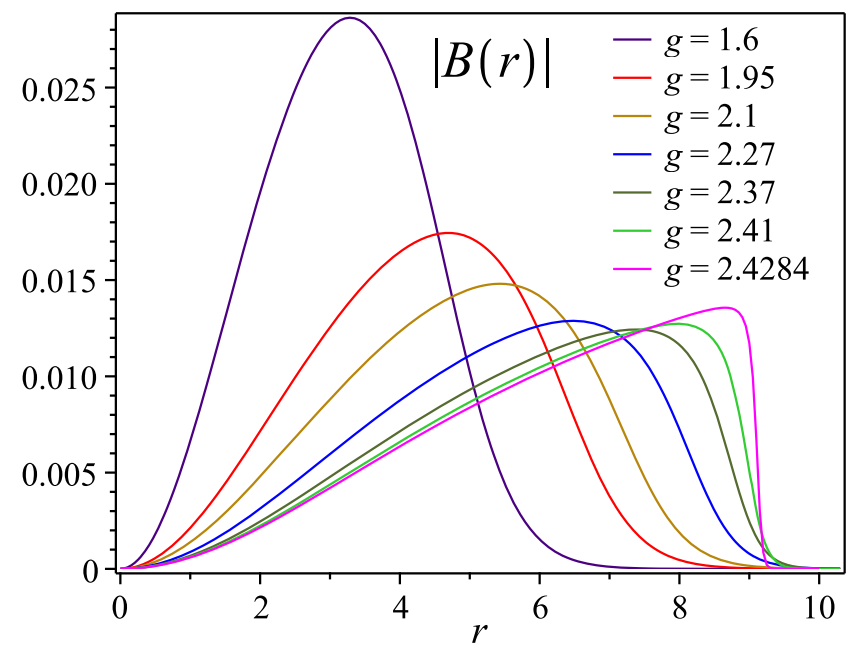

FIG. 9. Profiles of the magnetic field $B(r)$ with exponential decay engendered by the superpotential (94).

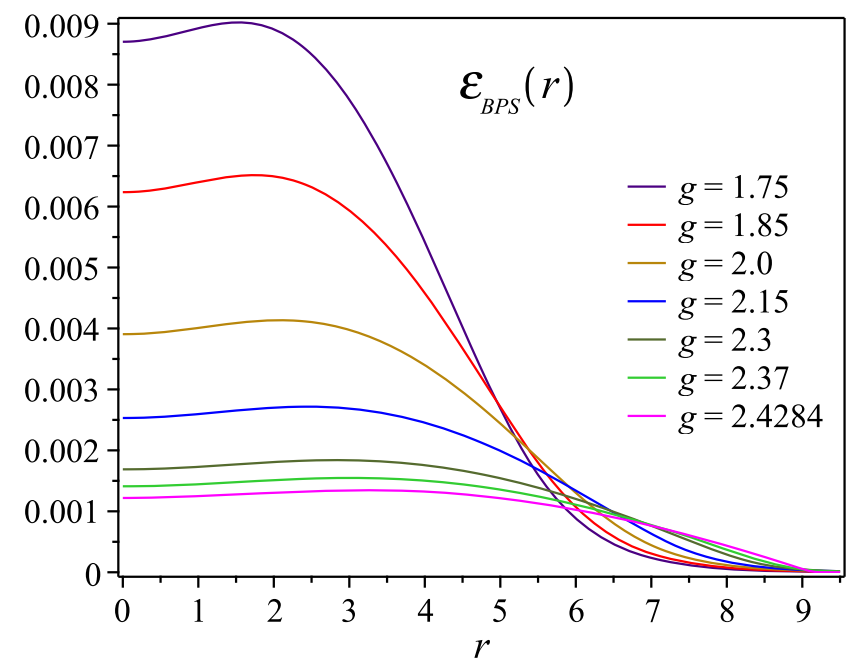

FIG. 10. Profiles of BPS energy density $\varepsilon_{\mathrm{BPS}}(r)$ with exponential decay engendered by the superpotential (94).

$$
\mathcal{U}(h)=\frac{\kappa^{4} W_{0}^{2}}{2 \lambda^{6} g^{6}}(1-h)^{2} .
$$

A similar potential was used in Ref. [28].

We performed our analysis by solving the BPS equations (63) and (64) by setting $N=1, \kappa=1, W_{0}=0.5$, $\lambda=1$, and various values of $g$. Figures 8,9 , and 10 present the profiles of the Skyrme and gauge field, the magnetic field and the BPS energy, respectively, for increasing values of $g$. For a sufficiently large value of $g$, we observe that all the profiles acquire a compactonlike structure.

The behaviors of the gauge vacuum value $a_{\infty}$ vs $g$ (red color) and the magnetic flux $|\Phi|$ vs $g$ (green color) depicted in Fig. 11 show a structure similar to the one observed in

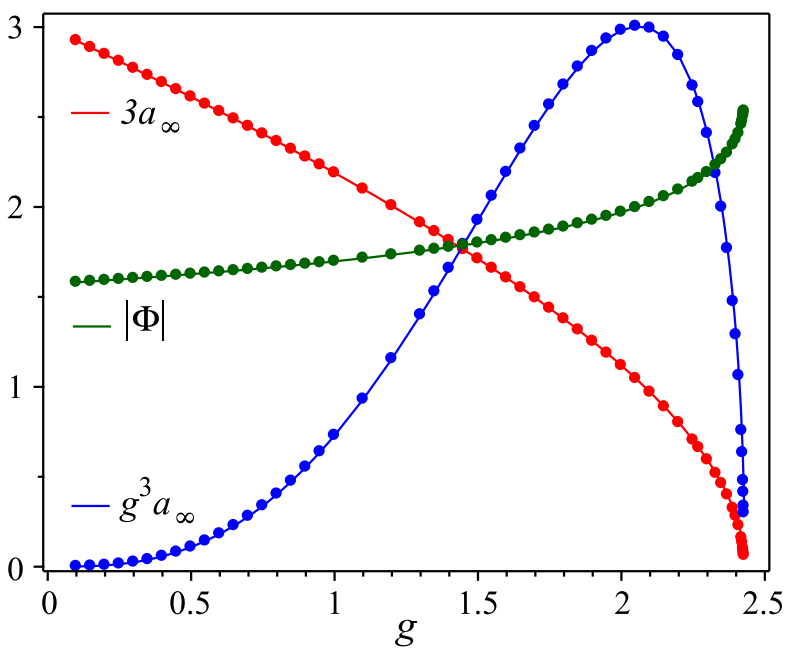

FIG. 11. Behaviors of the gauge vacuum value $a_{\infty}$ vs $g$ (red color), the magnetic flux $|\Phi|$ vs $g$ (green color), and the quantity $g^{3} a_{\infty}$ vs $g$ (blue color) of the solutions with exponential decay engendered by the superpotential (94). 


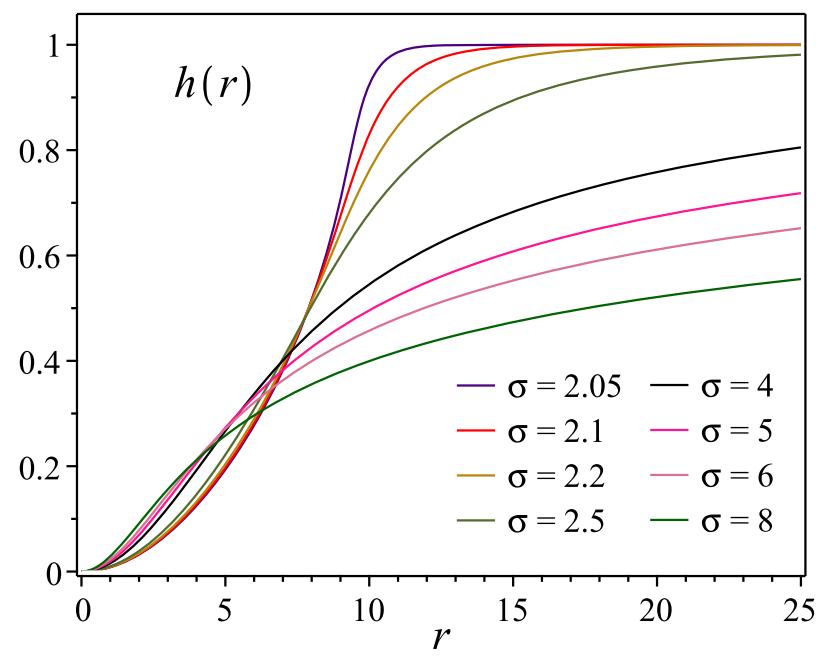

FIG. 12. The profiles $h(r)$ of the Skyrme field with power-law decay generated by the superpotential (96).

the compacton case. Similarly, we show the behavior of the quantity $g^{3} a_{\infty}$ vs $g$ (blue color), which controls the spread (85) of the solutions for sufficiently large values of $r$.

The numerical analysis shows that the gauge vacuum value $a_{\infty}$ only depends on the values of the electromagnetic coupling constant $g$ such it happens in the compacton case analyzed previously in Sec. VA.

\section{Solitons with power-law decay}

To obtain BPS solitons with power-law decay from solving the BPS equations (63) and (64), we consider the superpotential

$$
W(h)=W_{0}(1-h)^{\sigma},
$$

with $\sigma>2$, providing the potential

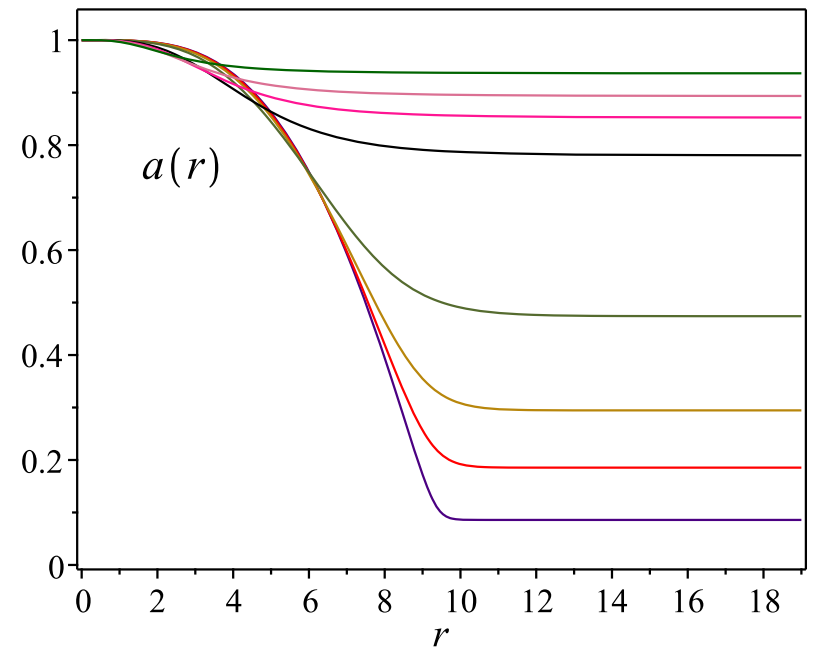

FIG. 13. The gauge field profiles $a(r)$ with power-law decay generated by the superpotential (96).

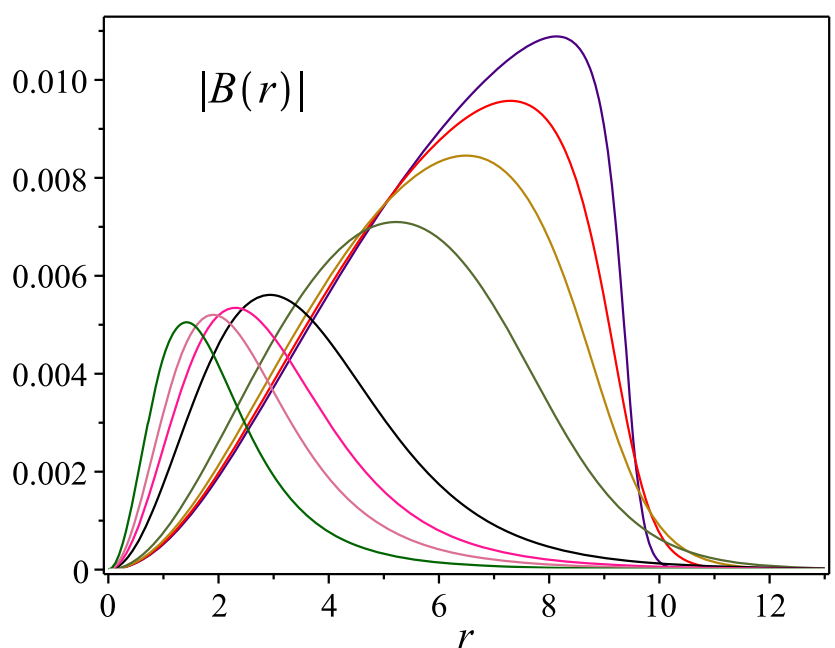

FIG. 14. The magnetic field profiles $B(r)$ with power-law decay generated by the superpotential (96).

$$
\mathcal{U}(h)=\frac{\kappa^{4} W_{0}^{2} \sigma^{2}}{8 \lambda^{6} g^{6}}(1-h)^{2(\sigma-1)} .
$$

We performed our analysis by solving the BPS equations (63) and (64) by setting $N=1, \kappa=1, W_{0}=0.5$, $\lambda=1, g=2.5$, and various values of the parameter $\sigma$. Figures 12, 13, 14, and 15 depict the profiles of the Skyrme field $h(r)$, the gauge field $a(r)$, the magnetic field $B(r)$, and the BPS energy density $\varepsilon_{\mathrm{BPS}}(r)$, respectively. The behavior of all the profiles becomes similar to a compactonlike form when the parameter $\sigma \rightarrow 2$.

For a fixed value of $\sigma>2$, similar to what happens in the two previous cases, presented in Secs. VA and V B, the numerical analysis has shown once again that the gauge vacuum value $a_{\infty}$ only depends on the electromagnetic coupling constant $g$. In addition, from Fig. 13, for a fixed value of $g$ and $\sigma \gg 2$, we have $a_{\infty} \rightarrow 1$ which implies the total magnetic flux $\Phi \rightarrow 0$ [see Eq. (67)].

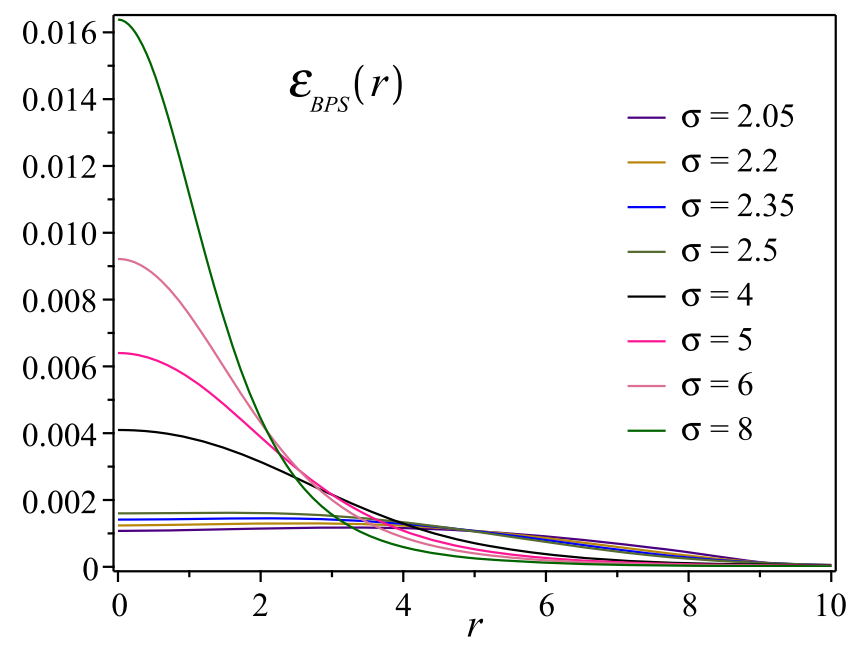

FIG. 15. The BPS energy density $\varepsilon_{\mathrm{BPS}}(r)$ with power-law decay generated by the superpotential (96). 


\section{CONCLUSIONS AND REMARKS}

We have shown the existence of BPS solitons in a type of generalized Chern-Simons baby Skyrme model (27) in which the generalized function $W\left(\phi_{n}\right)$ coupled to the sigma-model term becomes the superpotential that defines the self-dual potential (33). The guidelines for the construction of such a BPS model (25) or (27) are provided by the unsuccessful implementation of the BPS formalism in the Chern-Simons restricted baby Skyrme model introduced in Eq. (3). The successful implementation of the BPS formalism in the model (25) has allowed us to obtain an energy lower bound (BPS limit) and the self-dual equations satisfied by the field saturating such a limit. The BPS energy is proportional to the topological charge of the Skyrme field, so it is quantized. On the other hand, the total magnetic flux and total electric charge are proportional to each other but in general are not quantized. However, for sufficiently large values of the electromagnetic coupling constant $g$, both become quantized [see Eq. (93)].

The superpotential plays the principal role defining the BPS solitons; thus, we consider it being a well-behaved function in the whole target space. We have observed the existence of three classes of self-dual solutions closely related with the behavior of the superpotential. The first class of solitons we have obtained is the so-called compactons, which arise when the superpotential behaves like $W(r) \approx(1-h(r))^{\sigma}$ for $r \rightarrow R$ and $1<\sigma<2$, where $R$ is the compacton radius. The other two classes of solitons are noncompacton structures, i.e., they are regular functions in $0 \leq r<\infty$, but they are different because their respective tails have different behaviors for $r \rightarrow \infty$. Thus, the first noncompacton solitons are generated by a superpotential behaving like $W(r) \approx(1-h(r))^{2}$ for $r \rightarrow \infty$ of which the tail decays, following an exponential law $e^{-\alpha r^{2}}(\alpha>0)$. The second class of noncompacton solitons possesses a tail following a power-law decay $r^{-\beta}(\beta>0)$ for $r \rightarrow \infty$, and the superpotential behaves like $W(r) \approx(1-h(r))^{\sigma}$ with $\sigma>2$. Depending on the parameter values, the two last solitons can exhibit a compactonlike behavior.

It is important to emphasize the model proposed in the Lagrangian density (25) has a matching $\mathcal{N}=2$ SUSY extension possessing a self-dual or BPS structure, which is only possible through a set of auxiliary fields. In our context, the existence of such self-dual equations is ensured by the inclusion of the term proportional to $\left(\hat{n} \cdot D_{\mu} \vec{\phi}\right)^{2} W^{2}$ as in model (25). The superpotential $W$ is related to the target space area density and the Kähler metric of the sigma-model part [44]. Besides, the solutions of Eqs. (43) and (44) are type 1/4-BPS corresponding to the nontrivial phase of the $\mathcal{N}=2$ SUSY extension model.

We also are investigating the existence of BPS solitons in a baby Skyrme model gauged with the Maxwell-ChernSimons action and into the presence of Lorentz violation. The results will be reported elsewhere.

\section{ACKNOWLEDGMENTS}

This study was financed in part by the Coordenação de Aperfeiçoamento de Pessoal de Nível Superior-Brasil (CAPES), Finance Code 001. We thank also the Conselho Nacional de Desenvolvimento Científico e Tecnológico and the Fundação de Amparo à Pesquisa e ao Desenvolvimento Científico e Tecnológico do Maranhão (Brazilian government agencies). In particular, A.C. S., C.F.F., and A. L.M. are thankful for the full support from CAPES. R. C. acknowledges the support from Grants No. CNPq/306385/2015-5, No. CNPq/423862/ 2018-9, and No. FAPEMA/Universal-01131/17.
[1] T. H. R. Skyrme, Proc. R. Soc. A 260, 127 (1961); Nucl. Phys. 31, 556 (1962).

[2] I. Zahed and G. E. Brown, Phys. Rep. 142, 1 (1986).

[3] G. S. Adkins, C. R. Nappi, and E. Witten, Nucl. Phys. B228, 552 (1983); G. S. Adkins and C. R. Nappi, Nucl. Phys. B233, 109 (1984).

[4] O. V. Manko, N. S. Manton, and S. W. Wood, Phys. Rev. C 76, 055203 (2007); R. A. Battye, N. S. Manton, P. M. Sutcliffe, and S. W. Wood, Phys. Rev. C 80, 034323 (2009); P. H. C. Lau and N. S. Manton, Phys. Rev. Lett. 113, 232503 (2014); C. Halcrow, Nucl. Phys. B904, 106 (2016); C. J. Halcrow, C. King, and N. S. Manton, Phys. Rev. C 95, 031303(R) (2017).

[5] Y.-L. Ma, M. Harada, H. K. Lee, Y. Oh, B.-Y. Park, and M. Rho, Phys. Rev. D 88, 014016 (2013); 88, 079904(E) (2013); M. Harada, H. K. Lee, Y.-L. Ma, and M. Rho,
Phys. Rev. D 91, 096011 (2015); Y.-L. Ma and M. Rho, Sci. China Phys. Mech. Astron. 60, 032001 (2017).

[6] J. W. Holt, M. Rho, and W. Weise, Phys. Rep. 621, 2 (2016); C. Adam, C. Naya, J. Sanchez-Guillen, R. Vazquez, and A. Wereszczynski, Phys. Lett. B 742, 136 (2015); Phys. Rev. C 92, 025802 (2015); S. G. Nelmes and B. M. A. G. Piette, Phys. Rev. D 84, 085017 (2011); 85, 123004 (2012).

[7] C. Naya and P. Sutcliffe, Phys. Rev. Lett. 121, 232002 (2018).

[8] G. E. Volovik, Exotic Properties of Superfluid ${ }^{3} \mathrm{He}$ (World Scientific, Singapore, 1992); The Universe in a Helium Droplet (Oxford University, New York, 2009).

[9] S. D. Sarma and A. Pinczuk, Perspective in Quantum Hall Effects: Novel Quantum Liquids in Low-dimensional Semiconductor Structures (Wiley-VCH, Weinheim, 1996). 
[10] S. Mühlbauer, B. Binz, F. Jonietz, C. Pfleiderer, A. Rosch, A. Neubauer, R. Georgii, and P. Böni, Science 323, 915 (2009).

[11] S. D. Yi, S. Onoda, N. Nagaosa, and J. H. Han, Phys. Rev. B 80, 054416 (2009).

[12] X. Z. Yu, Y. Onose, N. Kanazawa, J. H. Park, J. H. Han, Y. Matsui, N. Nagaosa, and Y. Tokura, Nature (London) 465 , 901 (2010).

[13] U. Al Khawaja and H. Stoof, Nature (London) 411, 918 (2001).

[14] J. Fukuda and S. Žumer, Nat. Commun. 2, 246 (2011).

[15] G. Baskaran, arXiv:1108.3562.

[16] J. Garaud, J. Carlstrom, E. Babaev, and M. Speight, Phys. Rev. B 87, 014507 (2013).

[17] J. Garaud and E. Babaev, Sci. Rep. 5, 17540 (2015).

[18] A. A. Zyuzin, J. Garaud, and E. Babaev, Phys. Rev. Lett. 119, 167001 (2017).

[19] T. Winyard, M. Silaev, and E. Babaev, Phys. Rev. B 99, 024501 (2019).

[20] V. L. Vadimov, M. V. Sapozhnikov, and A. S. Mel'nikov, Appl. Phys. Lett. 113, 032402 (2018).

[21] A. N. Bogdanov, U. K. Rößler, M. Wolf, and K. H. Müller, Phys. Rev. B 66, 214410 (2002); U. K. Rößler, A. N. Bogdanov, and C. Pfleiderer, Nature (London) 442, 797 (2006); U. K. Rößler, A. A. Leonov, and A. N. Bogdanov, J. Phys. Conf. Ser. 303, 012105 (2011); C. J. Olson Reichhardt, S. Z. Lin, D. Ray, and C. Reichhardt, Physica (Amsterdam) 503C, 52 (2014).

[22] B. M. A. G. Piette, B. J. Schroers, and W. J. Zakrzewski, Z. Phys. C 65, 165 (1995); Nucl. Phys. B439, 205 (1995); R. A. Leese, M. Peyrard, and W. J. Zakrzewski, Nonlinearity 3, 773 (1990); B. M. A. G. Piette and W. J. Zakrzewski, Chaos Solitons Fractals 5, 2495 (1995); A. Kudryavtsev, B. M. A. G. Piette, and W. J. Zakrzewski, Eur. Phys. J. C 1, 333 (1998).

[23] T. Gisiger and M. B. Paranjape, Phys. Lett. B 384, 207 (1996).

[24] J. Gladikowski, B. M. A. G. Piette, and B. J. Schroers, Phys. Rev. D 53, 844 (1996).

[25] B. J. Schroers, Phys. Lett. B 356, 291 (1995).

[26] T. Gisiger and M. B. Paranjape, Phys. Rev. D 55, 7731 (1997).

[27] C. Adam, T. Romanczukiewicz, J. Sanchez-Guillen, and A. Wereszczynski, Phys. Rev. D 81, 085007 (2010); O. Alvarez, L. A. Ferreira, and J. Sanchez-Guillen, Nucl. Phys. B529, 689 (1998); Int. J. Mod. Phys. A 24, 1825 (2009); J. M. Speight, J. Phys. A 43, 405201 (2010).
[28] C. Adam, C. Naya, J. Sanchez-Guillen, and A. Wereszczynski Phys. Rev. D 86, 045010 (2012).

[29] C. Adam, J. Sanchez-Guillen, A. Wereszczynski, and W. J. Zakrzewski, Phys. Rev. D 87, 027703 (2013).

[30] C. Adam, C. Naya, T. Romanczukiewicz, J. SanchezGuillen, and A. Wereszczynski, J. High Energy Phys. 05 (2015) 155.

[31] L. Stepien, J. Phys. A 49, 175202 (2016).

[32] C. Adam and A. Wereszczynski, Phys. Rev. D 95, 116006 (2017).

[33] S. B. Gudnason, M. Nitta, and S. Sasaki, J. High Energy Phys. 02 (2016) 074.

[34] M. Nitta and S. Sasaki, Phys. Rev. D 91, 125025 (2015).

[35] C. Adam, J. M. Queiruga, J. Sanchez-Guillen, and A. Wereszczynski, J. High Energy Phys. 05 (2013) 108.

[36] C. Adam, T. Romanczukiewicz, M. Wachla, and A. Wereszczynski, J. High Energy Phys. 07 (2018) 097; M. Wachla, Phys. Rev. D 99, 065006 (2019).

[37] S. Deser, R. Jackiw, and S. Templeton, Ann. Phys. (N.Y.) 140, 372 (1982).

[38] G. V. Dunne, Aspects of Chern-Simons theory, in Les Houches Summer School in Theoretical Physics, Session 69: Topological Aspects of Low-dimensional Systems, edited by A. Comtet, T. Jolicoeur, S. Ouvry, and F. David (Springer-Verlag, Berlin, 2000).

[39] F. Wilczek, Fractional Statistics and Anyon Superconductivity (World Scientific, Singapore, 1990).

[40] S. M. Girvin, The Quantum Hall effect: Novel excitations and broken symmetries, in Les Houches Summer School in Theoretical Physics, Session 69: Topological Aspects of Low-dimensional Systems, edited by A. Comtet, T. Jolicoeur, S. Ouvry, and F. David (Springer-Verlag, Berlin, 2000).

[41] A. Yu. Loginov, J. Exp. Theor. Phys. 118, 217 (2014).

[42] C. Adam, C. Naya, J. Sanchez-Guillen, and A. Wereszczynski, J. High Energy Phys. 03 (2013) 012.

[43] A. Samoilenka and Ya. Shnir, Phys. Rev. D 95, 045002 (2017).

[44] J. M. Queiruga, J. Phys. A 52, 055202 (2019).

[45] S. Bolognesi and W. Zakrzewski, Phys. Rev. D 91, 045034 (2015).

[46] R. Casana, A. Cavalcante, and E. da Hora, J. High Energy Phys. 12 (2016) 051; R. Casana, E. da Hora, D. RubieraGarcia, and C. dos Santos, Eur. Phys. J. C 75, 380 (2015).

[47] E. Witten and D. Olive, Phys. Lett. B 78, 97 (1978).

[48] Z. Hlousek and D. Spector, Nucl. Phys. B370, 143 (1992). 